\title{
The prognosis factor of adjuvant radiation therapy after surgery in uterine sarcomas
}

\author{
This article was published in the following Dove Press journal: \\ OncoTargets and Therapy \\ 28 August 2015 \\ Number of times this article has been viewed
}

\author{
Hai-Ling Hou \\ Mao-Bin Meng \\ Xiu-Li Chen \\ Lu-Jun Zhao \\ Li Zhu \\ Bai-Lin Zhang \\ Ping Wang
}

Department of Radiation Oncology, CyberKnife Center, Key Laboratory of Cancer Prevention and Therapy, Tianjin Medical University Cancer Institute and Hospital, National Clinical Research Center for Cancer Tianjin, People's Republic of China

Correspondence: Ping Wang

Department of Radiation Oncology,

CyberKnife Center, Key Laboratory of Cancer Prevention and Therapy, Tianjin Medical University Cancer Institute and Hospital, National Clinical Research Center for Cancer, Huan-Hu-Xi Road, Ti-Yuan-Bei, He Xi District, Tianjin 300060, People's Republic of China Tel +86 22 2334 I405

Fax +8622 23344105

Email renqiu525@163.com
Objective: This retrospective study evaluated the role of adjuvant radiotherapy (AR) after surgery in patients with uterine sarcoma and analyzed the prognostic factors of local-regional failure-free survival (LRFFS) and overall survival (OS).

Patients and methods: A study of a total of 182 patients with uterine sarcoma was conducted between June 1994 and October 2014. Adjuvant radiotherapy was defined as postoperative external beam radiation to the pelvis (30-50 Gray/10-25 fractions at five fractions/week). The primary end point was LRFFS, and the secondary end point was OS. Kaplan-Meier curves were compared using the log-rank test. Cox regression analyses were used to determine prognosticators for LRFFS and OS.

Results: The median follow-up time of all patients was 75 months, with a 5-year LRFFS of $62.1 \%$. The 2-year and 5-year LRFFS rates were longer for those who received AR than for those who did not receive AR ( $83.4 \%$ vs $70.3 \% ; 78 \%$ vs $55.3 \% ; P=0.013)$. The 5 -year OS of all patients was $56.2 \%$, and no significant differences were observed in the 2-year and 5-year OS rates between these two groups $(82.7 \%$ vs $71.4 \% ; 64.1 \%$ vs $51.7 \% ; P=0.067)$. Importantly, in patients with leiomyosarcoma, the 2-year and 5-year LRFFS and OS rates were longer for those who received AR than for those who did not receive AR ( $P=0.04$ and $P=0.02$ for the 2-year and 5-year LRFFS, respectively).

Conclusion: Patients with uterine sarcoma who were treated with AR after surgery demonstrated an improved LRFFS compared with those who were treated with surgery alone, especially those patients with leiomyosarcoma. Therefore, the role of personalized adjuvant radiation for patients with uterine sarcoma still requires further discussion.

Keywords: uterine neoplasm, radiation, local-regional failure-free survival, overall survival

\section{Introduction}

Uterine sarcomas (USs) are rare tumors, constituting only approximately $3 \%$ of all uterine malignancies. ${ }^{1}$ The main pathological types include leiomyosarcoma (LMS), endometrial stromal sarcoma (ESS), and high-grade (undifferentiated) sarcoma of the endometrium. ${ }^{2}$ Although, in recent years, carcinosarcoma (CS) has been classified as a type of high-grade endometrial carcinoma, it has not yet been excluded in domestic and overseas studies of US. Although surgery is accepted as the primary treatment modality, the benefit of adjuvant radiotherapy (AR) remains unclear. The study reported here was a retrospective analysis of US cases from Tianjin Cancer Hospital (affiliated with Tianjin Medical University) from the last 20 years. The primary goal of this study was to analyze the impact of AR on outcome across the individual histologic subtypes. Significant prognostic factors for overall survival (OS) and local-regional failure-free survival (LRFFS) were also identified. 


\section{Patients and methods}

\section{Clinical data}

The study was approved by the Tianjin Medical University Cancer Hospital Ethics Committee. In all, 182 patients with US were admitted to Tianjin Medical University Cancer Institute and Hospital between June 1994 and October 2014. All of the patients were given a definitive pathological diagnosis. Among them, 79 cases were of LMS, 32 cases were of CS, 62 cases were of ESS, and nine cases were of adenosarcoma (AS). The patients were also classified by clinical stage according to the standard of the International Federation of Gynecology and Obstetrics (FIGO), as follows: 99 cases were at Stage I, 24 cases were at Stage II, 27 cases were at Stage III, and 32 cases were at Stage IV.

\section{Treatments}

Surgical procedures for newly diagnosed patients varied according to the different clinical stages and primarily consisted of hysterectomy/subtotal hysterectomy \pm excision of bilateral accessory \pm pelvic lymphadenectomy. Some patients had been treated for uterine fibroids and underwent local resections at other hospitals. However, their pathological results were indicative of US, and thus they underwent another radical surgery. Use of AR was defined as external beam radiation to the pelvis (including the postoperative tumor bed, vaginal cuff, and the corresponding lymphatic drainage area). A dosage of 30-50 Gray was given 10-25 times, five times per week. The clinical and pathologic characteristics of the patients are summarized in Tables 1 and 2.

\section{Statistical analysis}

Analysis was performed using SPSS software. Both OS and LRFFS were estimated using the Kaplan-Meier method, and individual treatment arms were compared using the log-rank method $(P<0.05)$. Univariate and multivariate analyses were performed using the Cox proportional hazards model. A two-side $P$-value $<0.05$ determined statistical significance for all tests.

\section{Results}

\section{LRFFS and OS}

The median follow-up time was 75.0 months. As of the end point, which was October 2014, a total of 90 patients had died. The 5-year LRFFS for all patients was $62.1 \%$, whereas the 2-year and 5-year LRFFS rates were $83.4 \%$ and $78 \%$, respectively, in the AR group, vs $70.3 \%$ and $55.3 \%$, respectively, in the No AR group ( $P=0.013)$. The total 5 -year OS was $56.2 \%$, whereas the 2 -year and 5 -year OS rates were $82.7 \%$ and $64.1 \%$, respectively, in the AR group, vs $71.4 \%$
Table I Patient and tumor characteristics

\begin{tabular}{ll}
\hline Characteristic & N (\%) \\
\hline Age, years & \\
$\quad \leq 50$ & $104(57.1)$ \\
$\quad>50$ & $78(42.9)$ \\
Menstrual state & \\
Premenopause & $52(28.6)$ \\
Perimenopause & $64(35.2)$ \\
Postmenopause & $65(35.7)$ \\
Histology & \\
LMS & $79(43.4)$ \\
CS & $32(17.6)$ \\
ESS & $62(34.1)$ \\
AS & $9(4.9)$ \\
Tumor grade & \\
Well differentiated & $76(41.8)$ \\
Moderately differentiated & $38(20.9)$ \\
Poorly differentiated & $68(37.4)$ \\
Surgical therapy & \\
TAH/SAH & $91(50.0)$ \\
TAH/SAH \pm BSO & $79(43.4)$ \\
TAH/SAH \pm BSO \pm pelvic exenteration & $12(6.6)$ \\
FIGO stage & \\
I & $99(54.4)$ \\
II & $24(13.2)$ \\
III & $27(14.8)$ \\
IV & $32(17.6)$ \\
Postoperative radiotherapy & \\
Yes & $49(26.9)$ \\
No & $133(73.1)$ \\
Yestoperative chemotherapy & $131(72.0)$ \\
No & $5 I(28.0)$ \\
\hline A6 & \\
\hline
\end{tabular}

Abbreviations: AS, adenosarcoma; BSO, bilateral salpingo-oophorectomy; CS, carcinosarcoma; ESS, endometrial stromal sarcoma; FIGO, International Federation of Gynecology and Obstetrics; LMS, leiomyosarcoma; SAH, subtotal abdominal hysterectomy; TAH, total abdominal hysterectomy.

and $51.7 \%$, respectively, in the No AR group $(P=0.067)$. The survival curves are shown in Figure 1A and B. In addition, the 5-year OS and LRFFS according to different histology are summarized in Table 3 .

\section{Patterns of failure}

Local recurrence was the major reason for failure. Before the last follow-up date, 59 patients experienced pelvic recurrence (32.4\%), including 18 cases with distant metastasis (9.9\%) and 21 cases with simple distant metastasis $(11.5 \%)$.

Table 2 Adjuvant radiotherapy (AR) administration by histology ( $n=182$ patients)

\begin{tabular}{lllll}
\hline Therapy & LMS, n (\%) & CS, n (\%) & ESS, n (\%) & AS, n (\%) \\
\hline AR & $16(20.3)$ & $12(37.5)$ & $17(27.4)$ & $4(44.4)$ \\
No AR & $63(79.7)$ & $20(62.5)$ & $45(72.6)$ & $5(55.6)$ \\
Total cases, n & 79 & 32 & 62 & 9 \\
\hline
\end{tabular}

Abbreviations: AS, adenosarcoma; CS, carcinosarcoma; ESS, endometrial stromal sarcoma; LMS, leiomyosarcoma. 

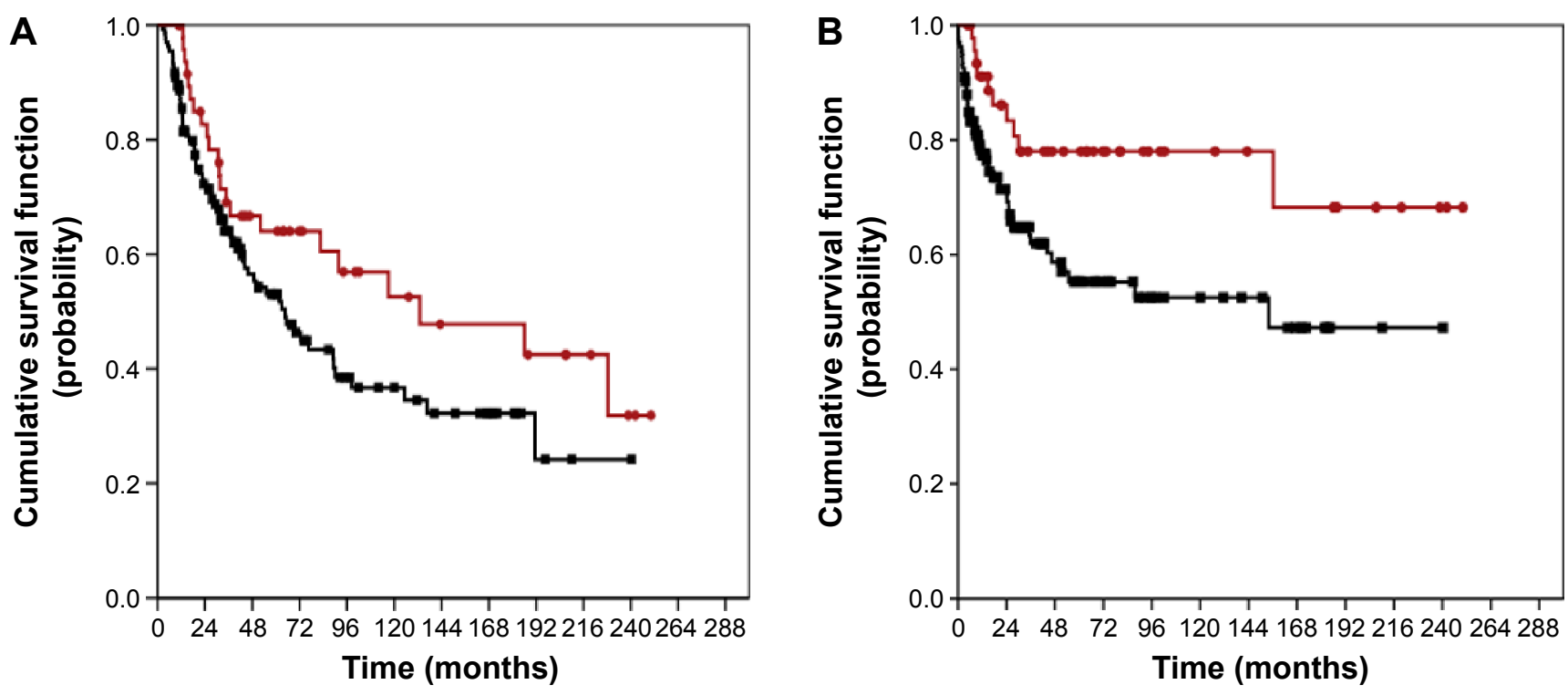

$\neg$ No AR $\neg$ AR $\quad$ NoAR-censored AR-censored

Figure I Actuarial overall survival (OS) and local-regional failure-free survival (LRFFS) of uterine sarcoma patients receiving definitive surgery with or without adjuvant radiotherapy (AR).

Notes: (A) OS; (B) LRFFS.

\section{Univariate analysis results of the LRFFS and OS}

Univariate analysis results showed that histology, tumor grade, stage, and treatment with postoperative radiotherapy were significant factors influencing LRFFS. Moreover, age, histology, tumor grade, and stage were significant factors influencing OS (Table 4).

\section{Multivariate analysis results for LRFFS and OS}

A Cox multivariate analysis showed that histology, tumor grade, stage, and treatment with postoperative radiotherapy were significant factors influencing LRFFS, whereas age, histology, tumor grade, and stage were significant factors influencing OS (Table 4).

\section{Subgroup analysis}

Subgroup analysis showed benefit for LRFFS and OS in patients with LMS, with a 5 -year LRFFS of $78.7 \%$ vs $44 \%$
( $P=0.037)$, and a 5 -year OS of $71.8 \%$ vs $40.2 \%(P=0.018)$, in favor of AR (Figure 2A and B). There was no significant difference in patients with CS (Figure 2C and D) and ESS (Figure 2E and F) based on whether they received AR. Small patient numbers in the AS group precluded any statistical comparisons.

\section{Discussion}

According to the National Comprehensive Cancer Network guidelines, ${ }^{3}$ the principal treatment for US is surgery; however, the application of postoperative adjuvant therapy is widely debated among gynecologic and radiation oncologists. The study reported here was a retrospective analysis of 182 patients with US from our hospital. The 5-year OS of 56.2\% is consistent with other large studies, which have reported 5 -year survival rates of $31 \%-56 \% .^{4-8}$ In the current study, postoperative radiotherapy grants no benefit in terms of the total survival rate, but it can obviously improve the local control rate. Compared to surgery alone, AR can improve the 5-year

Table 3 The 5-year overall survival (OS) and local-regional failure-free survival (LRFFS) by histology

\begin{tabular}{|c|c|c|c|c|c|c|}
\hline \multirow[t]{2}{*}{ Tumor type } & \multicolumn{3}{|c|}{ OS, $\%$} & \multicolumn{3}{|c|}{ LRFFS, \% } \\
\hline & AR & No AR & $P$-value & $A R$ & No AR & $P$-value \\
\hline LMS & 71.8 & 40.2 & 0.018 & 78.7 & 44.0 & 0.037 \\
\hline CS & 55.0 & 29.5 & 0.150 & 72.9 & 53.1 & 0.280 \\
\hline ESS & 67.0 & 85.8 & 0.390 & 76.9 & 73.1 & 0.810 \\
\hline
\end{tabular}

Abbreviations: AR, adjuvant radiotherapy; CS, carcinosarcoma; ESS, endometrial stromal sarcoma; LMS, leiomyosarcoma. 
Table 4 Prognostic factors for local-regional failure-free survival (LRFFS) and overall survival (OS): univariate and multivariate analyses

\begin{tabular}{|c|c|c|c|c|}
\hline \multirow[t]{3}{*}{ Factor } & \multicolumn{4}{|c|}{$P$-value } \\
\hline & \multicolumn{2}{|c|}{ Univariate } & \multicolumn{2}{|c|}{ Multivariate } \\
\hline & os & LRFFS & OS & LRFFS \\
\hline Age & 0.0130 & 0.0080 & 0.0130 & 0.2690 \\
\hline Menstrual states & 0.1680 & 0.2310 & 0.0880 & 0.5570 \\
\hline Histology & 0.0030 & 0.0030 & 0.0070 & 0.0070 \\
\hline Tumor grade & 0.0001 & 0.0001 & 0.0001 & 0.0001 \\
\hline Type of surgery & 0.1060 & 0.1250 & 0.7020 & 0.6860 \\
\hline FIGO stage & 0.0001 & 0.0001 & 0.0001 & 0.0001 \\
\hline Postoperative radiotherapy & 0.0670 & 0.0370 & 0.0670 & 0.0130 \\
\hline Postoperative chemotherapy & 0.0760 & 0.0580 & 0.0760 & 0.0970 \\
\hline
\end{tabular}

Abbreviation: FIGO, International Federation of Gynecology and Obstetrics.

LRFFS from $55.3 \%$ to $78.0 \%$. More importantly, the subset analysis showed that, for LMS, postoperative radiotherapy can not only reduce the local recurrence rate but also improve survival. Other histology failed to show statistically significant results, which may be due to the limited number of cases.

Due to the low incidence of US, reports about the application of auxiliary radiation in the treatment of US are also limited. The previously reported studies are mainly retrospective in nature and primarily include patients with early LMS and CS. There is a lack of rigorous prospective randomized controlled studies. Most current research suggests that auxiliary radiotherapy can improve the control rate of local lesions and reduce the local recurrence of US, but that it has no effects on the survival rate. ${ }^{9}$

Wright et al studied 1,898 cases of CS and 1,088 cases of LMS (all at Stage I/II) and found that for patients with CS, postoperative radiotherapy could reduce the risk of death by $21 \%{ }^{10}$ Postoperative radiotherapy can the reduce risk of death by $25 \%$ especially in patients without lymph node dissection. For patients with LMS, postoperative radiotherapy did not improve the OS rate.

Sampath et $\mathrm{al}^{11}$ conducted a retrospective study on 3,650 patients with US and also concluded that radiation therapy failed to improve the total survival rate; a further analysis showed that for the 2,206 patients who underwent radical surgery as the initial treatment, the 5-year LRFFS rates were $93 \%$ and $85 \%$ in the radiotherapy group and nonradiotherapy group, respectively $(P<0.001)$. Regardless of the pathological type, postoperative radiotherapy can reduce the local recurrence rate; however, the limitation of Sampath et al's study was that the staging of the 1,366 cases was not reported, which may have impacted the results.

Reed et $\mathrm{al}^{12}$ performed a prospective, randomized controlled trial during 1988-2001. The recurrence rates and the survival conditions of the 222 US patients at Stage I/II were observed, including 103 cases of LMS, 91 of CS, and 28 cases of ESS. The patients were randomly divided into the observation group and the radiotherapy group. The effects of pelvic radiotherapy were then compared between the groups. The results showed that the median LRFFS rates were 6.22 and 4.93 years in the radiotherapy group and the observation group, respectively $(P=0.35)$. The median OS rates were 8.53 and 6.78 years in the radiotherapy and the observation group, respectively ( $P=0.92)$, whereas the 5 -year local recurrence rates were $18.8 \%$ and $35.9 \%$ in the radiotherapy and the observation group, respectively $(P=0.0013)$.

More importantly, previous subset studies that were based on pathological type showed that for LMS, radiation therapy failed to improve the local control rate and the overall survival rate. ${ }^{12}$ In regards to $\mathrm{CS}$, postoperative radiotherapy could reduce the local recurrence rate from $47 \%$ to $24 \% .{ }^{12}$ Additionally, in the current study, the subgroup analysis demonstrated that postoperative radiotherapy conferred benefits for both the local control rates and the survival rates of patients with LMS. This result is not in accordance with most previous reports, which may be due to the smaller number of cases. Among the 79 patients with LMS, only 16 received radiotherapy; thus, more cases need to be included for further analysis. As far as we are aware, there are even fewer related studies that include ESS and AS, which have a lower incidence, and most of these are retrospective studies of fewer than 50 cases. ${ }^{13-16}$ It is worth mentioning that a retrospective study reported by Barney et $\mathrm{al}^{17}$ of 1,010 patients with ESS showed that the 5-year survival rates were $72.2 \%$ and $83.2 \%$ in the surgery plus radiotherapy group and the simple surgery group, respectively. Moreover, factors that indicated a poor prognosis included FIGO staging, histological grade and age. Postoperative radiation therapy did not improve survival rate. However, the study did not report the data related to local recurrence. 


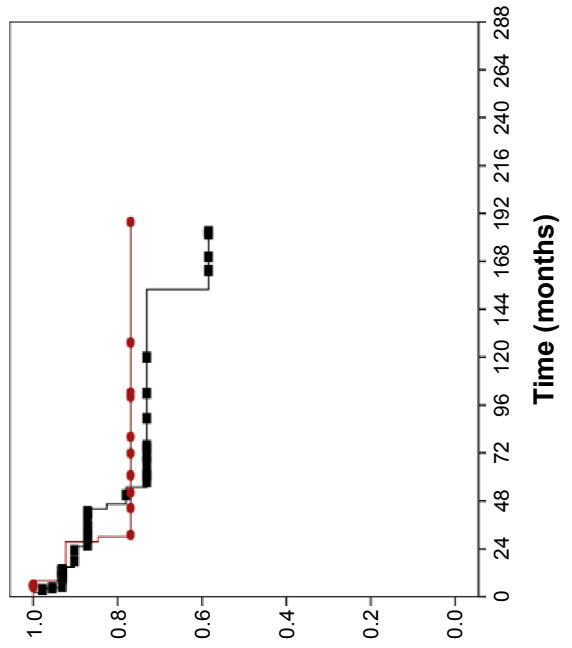

(Kł!!!qeqoud)

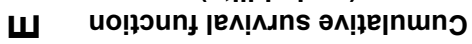

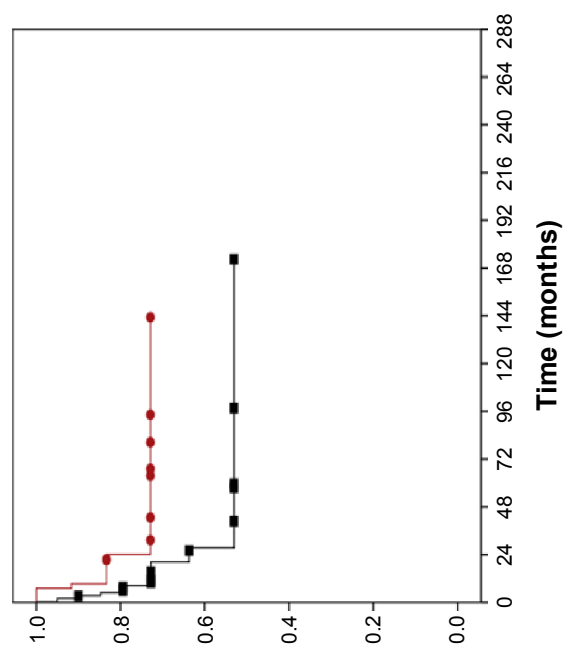

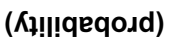

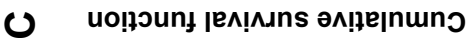

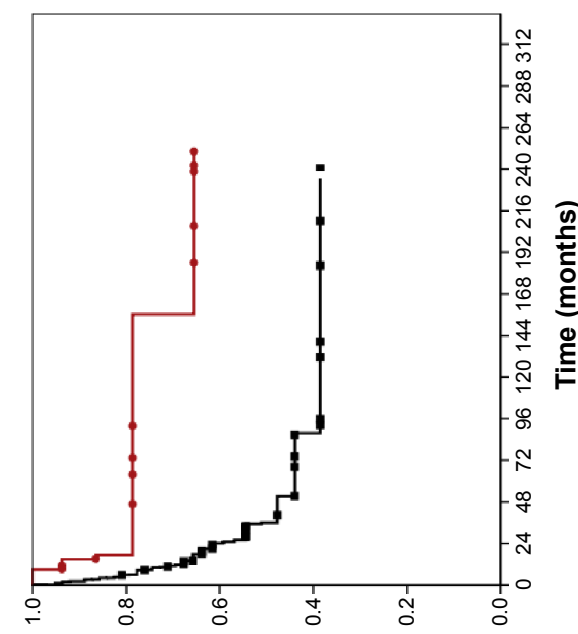

( $\left.\kappa_{+! ! ! q e q o, d}\right)$

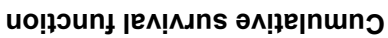

$<$

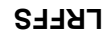

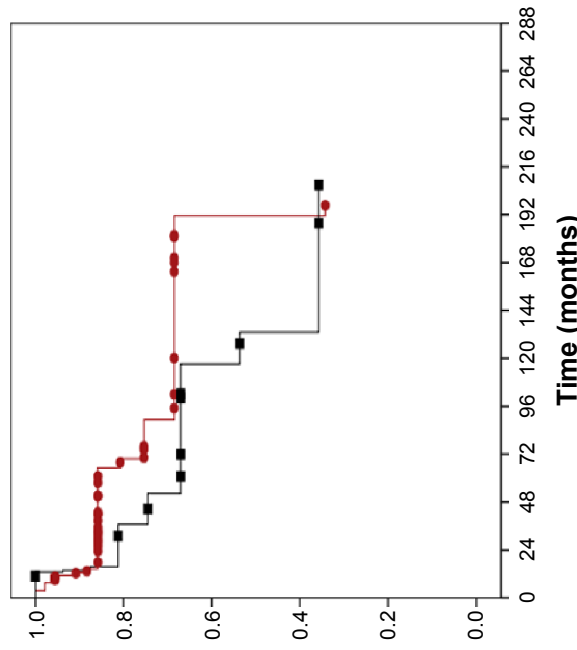

(Kt!!!qeqo.dd)

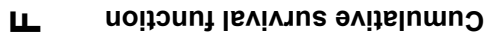

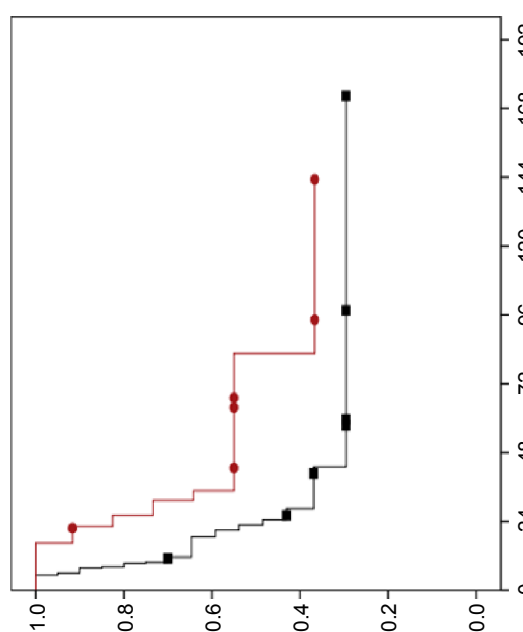

(K!!!!qeqoud)

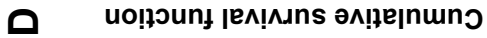

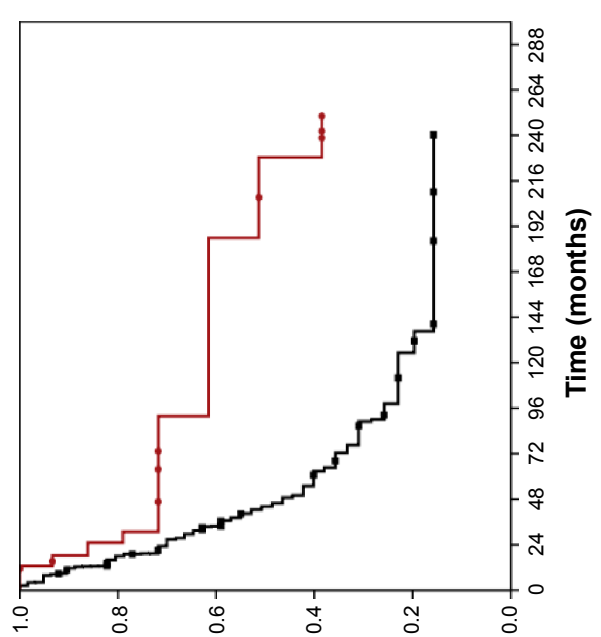

(К‼!qeqosd)

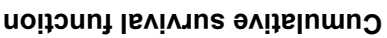

so 


\section{Conclusion}

It is difficult to define the role for AR after definitive surgery because of the low incidence, heterogeneous histologic subtypes, and lack of validated prospective data in USs. We conclude that AR can significantly improve the LRFFS of patients with US and can increase the OS and LRFFS of patients with LMS. However, a prospective study and a subset analysis on a larger sample size should be conducted to select relevant prognostic factors and to provide evidence for postoperative individualized auxiliary radiotherapy for US.

\section{Acknowledgment}

This work was supported by the National Natural Science Foundation of China (numbers 81372518 and 81201754). No benefits in any form have been or will be received from a commercial party directly or indirectly related to the subject of this article.

\section{Disclosure}

All authors read and approved the manuscript and have no financial disclosures. No copyrighted information, patient photographs, identifiers, or other protected health information is used in this paper. No text, text boxes, figures, or tables in this article have been previously published or are owned by another party.

Other than the funding described, the authors declare no conflicts of interest in this work.

\section{References}

1. D'AngeloE,PratJ.Uterine sarcomas: areview. GynecolOncol.2010;116(1): 131-139.

2. El-Khalfaoui K, du Bois A, Heitz F, Kurzeder C, Sehouli J, Harter P. Current and future options in the management and treatment of uterine sarcoma. Ther Adv Med Oncol. 2014;6(1):21-28.

3. Grimer R, Judson I, Peake D, Seddon B. Guidelines for the management of soft tissue sarcomas. Sarcoma. 2010;2010:506182.
4. Oláh KS, Gee H, Blunt S, Dunn JA, Kelly K, Chan KK. Retrospective analysis of 318 cases of uterine sarcoma. Eur J Cancer. 1991;27(9): 1095-1099.

5. Major FJ, Blessing JA, Silverberg SG, et al. Prognostic factors in earlystage uterine sarcoma. A Gynecologic Oncology Group study. Cancer. 1993;71(4 Suppl):1702-1709.

6. Callister M, Ramondetta LM, Jhingran A, Burke TW, Eifel PJ. Malignant mixed Müllerian tumors of the uterus: analysis of patterns of failure, prognostic factors, and treatment outcome. Int J Radiat Oncol Biol Phys. 2004;58(3):786-796.

7. Sorbe B, Johansson B. Prophylactic pelvic irradiation as part of primary therapy in uterine sarcomas. Int J Oncol. 2008;32(5):1111-1117.

8. Moskovic E, MacSweeney E, Law M, Price A. Survival, patterns of spread and prognostic factors in uterine sarcoma: a study of 76 patients. Br J Radiol. 1993;66(791):1009-1015.

9. Gao Kun, Li Li. Postoperative adjuvant radiotherapy in the treatment of uterine sarcomas. Journal of Practical Obstetrics and Gynecology. 2012; $28: 13-15$

10. Wright JD, Seshan VE, Shah M, et al. The role of radiation in improving survival for early-stage carcinosarcoma and leiomyosarcoma. $\mathrm{Am}$ J Obstet Gynecol. 2008;199(5):536.e1-e8.

11. Sampath S, Schultheiss TE, Ryu JK, Wong JY. The role of adjuvant radiation in uterine sarcomas. Int J Radiat Oncol Biol Phys. 2010;76(3): 728-734.

12. Reed NS, Mangioni C, Malmström H, et al; European Organisation for Research and Treatment of Cancer Gynaecological Cancer Group. Phase III randomised study to evaluate the role of adjuvant pelvic radiotherapy in the treatment of uterine sarcomas stages I and II: an European Organisation for Research and Treatment of Cancer Gynaecological Cancer Group Study (protocol 55874). Eur J Cancer. 2008;44(6):808-818.

13. Bodner K, Bodner-Adler B, Obermair A, et al. Prognostic parameters in endometrial stromal sarcoma: a clinicopathologic study in 31 patients. Gynecol Oncol. 2001;81(2):160-165.

14. Gadducci A, Sartori E, Landoni F, et al. Endometrial stromal sarcoma: analysis of treatment failures and survival. Gynecol Oncol. 1996;63(2): 247-253.

15. Leath CA 3rd, Huh WK, Hyde J Jr, et al. A multi-institutional review of outcomes of endometrial stromal sarcoma. Gynecol Oncol. 2007;105(3): 630-634.

16. Weitmann HD, Knocke TH, Kucera H, Pötter R. Radiation therapy in the treatment of endometrial stromal sarcoma. Int J Radiat Oncol Biol Phys. 2001;49(3):739-748.

17. Barney B, Tward JD, Skidmore T, Gaffney DK. Does radiotherapy or lymphadenectomy improve survival in endometrial stromal sarcoma? Int J Gynecol Cancer. 2009;19(7):1232-1238.
OncoTargets and Therapy

\section{Publish your work in this journal}

OncoTargets and Therapy is an international, peer-reviewed, open access journal focusing on the pathological basis of all cancers, potential targets for therapy and treatment protocols employed to improve the management of cancer patients. The journal also focuses on the impact of management programs and new therapeutic agents and protocols on

\section{Dovepress}

patient perspectives such as quality of life, adherence and satisfaction. The manuscript management system is completely online and includes a very quick and fair peer-review system, which is all easy to use. Visit http://www.dovepress.com/testimonials.php to read real quotes from published authors. 